\title{
Perfil Epidemiológico de Pacientes Portadores de Doença Renal Crônica Terminal em Programa de Hemodiálise em Clínica de Santa Cruz do Sul - RS
}

\author{
Aglaupe Ferreira Bonfim Pereira ${ }^{1}$, Cássia Pinheiro Kapper ${ }^{1}$, \\ Gustavo Biondo ${ }^{1}$, Maura David ${ }^{1}$, Victoria Accioly Russowsky ${ }^{1}$, \\ Homero Agra ${ }^{1,2}$
}

\section{Resumo}

A DRC é definida por lesão renal e perda progressiva e irreversível da função renal com taxa de filtração glomerular (TFG) $<60 \mathrm{ml} / \mathrm{min} / 1,73 \mathrm{~m}^{2}$ por período $\geq 3$ meses, com ou sem lesão renal. $\mathrm{O}$ dano renal é detectado pela presença de albuminúria $\geq 30 \mathrm{mg} /$ dia. A DRC evolui do estágio 1 ao 5 até atingir TFG de $10 \mathrm{ml} / \mathrm{min}$ e sintomas urêmicos. No estágio 5 o paciente necessita de terapia renal de substituição (TRS), sendo hemodiálise (HD) ou diálise peritoneal ambulatorial contínua (CAPD). Os grupos de risco para DRC são hipertensos, diabéticos e pacientes com história familiar de DRC. Dentre as causas de DRC, destacam-se a nefroesclerose hipertensiva e glomerulopatia diabética. O objetivo desse estudo foi verificar as principais etiologias de DRC dialítica em clínica de diálise do interior do Rio Grande do Sul.

Foi realizado um estudo transversal de dados referentes aos pacientes de hemodiálise. Os dados foram coletados através do banco de informações nefrossystem, tabulados e submetidos à análise estatística descritiva simples de acordo com as principais etiologias da DRC dos pacientes em diálise.

Dos 110 pacientes analisados, 64 são homens e 67 possuem idade superior a 60 anos, $100(90,90 \%)$ dos pacientes apresentam fístula arteriovenosa como o tipo de acesso para hemodiálise. Em relação à doença de base observa-se que $39(35,45 \%)$ pacientes apresentam nefropatia diabética, enquanto que 26 $(23,63 \%)$ apresentam nefropatia hipertensiva, seguido de $15,45 \%$ com doença de origem indeterminada, 9,09\% glomerulonefrite crônica, 7,27\% pielonefrite crônica, $4,54 \%$ doença renal policística e $2,72 \%$ apresentando 
nefropatia lúpica. Dos 110 pacientes, 99 tiveram o ktv estimado e 93 $(93,94 \%)$ obtiveram a meta atingida $(\mathrm{kt} / \mathrm{v}>1,20)$.

Observamos que dados sobre DRC do centro de referência se assemelham com dados mundiais, indicando a nefropatia diabética como principal causa de perda da função renal, seguida da hipertensiva. O envelhecimento da população, os hábitos sedentários e a dieta inadequada tendem a elevar o número de doentes renais. O reconhecimento de pacientes com fatores de risco é essencial para diagnóstico e abordagem terapêutica precoce melhorando o prognóstico.

Palavras Chave: Doença renal crônica/etiologia, Doença renal crônica dialítica, Taxa de filtração glomerular.

\footnotetext{
${ }^{1}$ Acadêmico do Curso de Medicina, Universidade de Santa Cruz do Sul - UNISC, Santa Cruz do Sul-RS

${ }^{2}$ Nefrologista, professor do Curso de Medicina, Universidade de Santa Cruz do Sul - UNISC, Santa Cruz do Sul - RS
}

\section{Introdução}

A Doença Renal Crônica (DRC) é um problema de saúde mundial, sendo denominada "Epidemia do novo Milênio". Dados do estudo de Framingham sugerem que aproximadamente 20 milhões de pessoas nos EUA estão em risco para DRC. No Brasil estes dados apontam para 12 milhões de pessoas sob o mesmo risco.

A definição de DRC claramente estabelecida está baseada em três pilares: em primeiro, um componente anatômico ou estrutural como marcadores de lesão renal; em segundo, o componente funcional renal, baseado na taxa de filtração glomerular (TFG), que nada mais é do que a capacidade dos rins de eliminar uma substância do sangue; em terceiro, um componente temporal, por um período de pelo menos três meses. A DRC é definida, então, por lesão renal e perda progressiva e irreversível da função dos rins com taxa de filtração glomerular (TFG) $<60 \mathrm{ml} / \mathrm{min} / 1,73 \mathrm{~m}^{2}$ por período $\geq 3$ meses, com ou sem lesão renal. O dano renal é geralmente detectado pela presença de albuminúria $\geq 30 \mathrm{mg} / \mathrm{dia}$ ou alterações equivalentes.

É classificada em estágios de acordo com a função renal, independente do diagnóstico etiológico. São 5 estágios, em ordem decrescente de função renal. No estágio 1, ocorre lesão renal com filtração glomerular preservada, acima de $90 \mathrm{ml} / \mathrm{min}$. A DRC crônica evolui, piorando taxas de filtração glomerular até atingir o estágio 5, com sintomas urêmicos e uma TFG em torno de $10 \mathrm{ml} / \mathrm{min}$, diagnosticado com DRC terminal (DRCT). 
Nesse estágio o paciente necessita de terapia renal de substituição (TRS) que compreendem hemodiálise (HD) ou diálise peritoneal ambulatorial contínua (CAPD).

A doença sempre inicia em uma fase de função renal normal ainda sem lesão renal. Este estágio inicial pré-doença compreende um grande valor epidemiológico por se tratar de pacientes com fatores de risco para estabelecimento da doença mas que ainda não desenvolveram lesão renal. Esses fatores de risco conferem a determinados grupos de pacientes maior suscetibilidade para DRC, sendo considerados grupos de risco. Sao eles: hipertensos, diabéticos, idosos, pacientes com doenca cardiovascular e com história familiar de DRC.

Dentre as causas de DRC, destacam-se a nefroesclerose hipertensiva e glomerulopatia diabética, consideras atualmente, as principais etiologias de lesão renal irreversível no Brasil. Outras etiologias possíveis são as glomerulopatias primárias e a doença renal policística, além da idiopática.

A DRC é uma patologia de custo e tratamento elevado. Portanto, é importante avaliar as suas principais etiologias, de modo a evitar que os pacientes cheguem à fase terminal da doença, gerando gastos com saúde. Com o aumento de diagnósticos de DRC em nível mundial, percebe-se a necessidade de conhecer suas causas para que possam ser adequadamente manejadas, a fim de evitar que os pacientes cheguem na DRCT necessitando de diálise, melhorando a qualidade de vida e diminuindo os gastos públicos com saúde. Desse modo, o objetivo desse estudo foi verificar as principais etiologias de DRC dialítica em clínica de diálise do interior do Rio Grande do Sul.

\section{Metodologia}

Foi realizado um estudo transversal de análise dos dados referentes aos pacientes que se encontram em programa de hemodiálise em um centro de referência na cidade de Santa Cruz do Sul, Rio Grande do Sul. Os dados foram coletados no mês de setembro de 2015. Tais dados foram obtidos através do banco de informações do programa nefrossystem, sendo avaliadas as principais etiologias da doença renal crônica dos pacientes em diálise. Os dados foram tabulados e submetidos à análise estatística descritiva simples, utilizando o Software Microsoft Excel.

\section{Resultados}

Dos 110 pacientes analisados, $64(58,19 \%)$ são homens e $67(60,90 \%)$ possuem idade superior a 60 anos, $100(90,90 \%)$ dos pacientes apresentam fístula arteriovenosa como o tipo de acesso para hemodiálise. Em relação à doença de base observa-se que $39(35,45 \%)$ pacientes apresentam nefropatia diabética, enquanto que $26(23,63 \%)$ apresentam nefropatia hipertensiva, seguido de $15,45 \%$ apresentando doença de origem indeterminada, $10(9,09 \%)$ glomerulonefrite crônica, 8 (7,27\%) pielonefrite crônica, 5 $(4,54 \%)$ doença renal policística e $3(2,72 \%)$ apresentando nefropatia lúpica. A diálise para ser adequada precisa fornecer um $\mathrm{kTv}>1,20$ e esse índice corresponde a uma 
estimativa entre a uréia pré e pós hemodiálise. Os pacientes que possuem o kTv examinado precisam estar em diálise por um período superior a 3 meses. Dos 110 pacientes, somente 99 tiveram o ktv estimado e 93,94\% obtiveram a meta atingida (kt/v $>1,20)$. Além do kTv, a adequação da hemodiálise é avaliada através de indicadores como o estadiamento de doença óssea, anemia e nutrição.

\section{Conclusões}

A DRC corresponde a uma doença de âmbito mundial, sendo sua principal causa a nefropatia diabética e hipertensiva. É uma doença insidiosa e assintomática no maior tempo de sua evolução, e a sua progressão é avaliada através da taxa de filtração glomerular (TFG), sendo diversos fatores associados à sua etiologia e prognóstico relacionado à perda de função renal. É importante reconhecer os pacientes que estão em risco de desenvolver a DRC, com objetivo de um diagnóstico e abordagem terapêutica precoce. A DRC tem sido descrita como um dos principais determinantes de risco de eventos cardiovasculares, somada aos fatores de risco tradicionais (como DM e HAS) para DCV que constitui hoje a principal causa de morte no mundo. De acordo com o Censo Brasileiro de Diálise publicado em 2012, o número de pacientes com DRCT no Brasil praticamente duplicou na última década, de modo que em 2000 havia 42.695 e no ano de 2011 atingiu 91.314 indivíduos. Atualmente os métodos de hemodiálise são mais seguros, visto que as máquinas hemodialisadoras possuem monitores de detecção de osmolaridade, temperatura, hemoglobina e pressão arterial e venosa.

Dados sobre DRC do centro de referência apresentam semelhança com dados mundiais, indicando a nefropatia diabética como a principal causa de perda da função renal, seguida da hipertensiva. Observa-se aumento em nível mundial de diabéticos e hipertensos devido ao envelhecimento da população, aos hábitos de vida sedentários e dieta inadequada. $\mathrm{O}$ diagnóstico precoce, encaminhamento imediato aos centros de referência e a adoção de medidas para interromper a progressão da DRC estão entre as estratégias-chave para melhorar os desfechos e, em alguns casos, evitar a hemodiálise.

Apesar do aumento considerável da DRC, a prevalência de pacientes em diálise no Brasil está abaixo de nações com perfil semelhante, apontando para a necessidade de identificação e tratamento adequado dos pacientes com fatores de risco para a doença, bem como, seu diagnóstico precoce e tratamento, visando o cuidado integral desses pacientes, tendo como principais objetivos a redução de desfechos desfavoráveis, como a mortalidade cardiovascular e a progressão para DRCT.

\section{Referências}

KIRSZTAJN, G. M.; SOUZA, E.; ROMÃO, JR, J. E.; BASTOS, M. G.; MEYER, F.; ANDRADA, N. C. Doença Renal Crônica (Pré-terapia Renal Substitutiva): Diagnóstico. Projeto diretrizes - Associação Médica Brasileira, 2011. 
MOURA, L. R. R. Insuficiência renal. São Paulo, SP: Sociedade Brasileira de Nefrologia, 17 set. 2013. Disponível em: <http://www.sbn.org.br/leigos/index.php?insuficienciaRenal>

Ministério da Saúde (BR).; Secretaria de Atenção à Saúde.; Departamento de Atenção Especializada e Temática. Diretrizes Clínicas para o Cuidado ao Paciente com Doença Renal Crônica - DRC no Sistema Único de Saúde. Brasília: Ministério da Saúde, 2014.

LEVEY, A. S.; ROCCO, M. V.; ANDERSON, S.; ANDREOLI, S. P.; et al. K/DOQI Clinical Practice Guidelines for Chronic Kidney Disease: Evaluation, Classification and Stratification, In :American Journal of Kidney Diseases. 43, 5 SUPPL. 1. 2004. 